\title{
Impacto de mudanças climáticas sobre a distribuição geográfica potencial de Ilex paraguariensis
}

\author{
Impact of climate change on the potential \\ geographical distribution of Ilex paraguariensis
}

Mariéle Alves Ferrer da Silva ${ }^{1}$, Pedro Higuchi ${ }^{1,2}$ \& Ana Carolina da Silva ${ }^{1}$

\begin{abstract}
Resumo
Foi modelado o nicho climático e avaliamos o impacto de mudanças climáticas sobre a distribuição geográfica potencial de Ilex paraguariensis. Esta espécie apresenta elevada importância sociocultural, econômica e ecológica na porção sul da Floresta Atlântica. As áreas de ocorrência foram definidas por meio das coordenadas geográficas das localidades onde a espécie foi registrada. Foram consideradas oito variáveis climáticas obtidas a partir da base de dados do WorldClim. A modelagem do nicho climático da espécie foi realizada por meio de dez algoritmos. A predição da área de adequabilidade climática, contemporânea e futura (2070) da espécie, foi realizada a partir do consenso dos ajustes com boa performance (TSS > 0,40). Para predições futuras, foram considerados cenários de mudanças climáticas otimista (RCP4.5) e pessimista (RCP8.5), com base no quinto relatório do IPCC. Verificou-se que a espécie ocorre, predominantemente, no domínio Atlântico. Enquanto que no cenário otimista, I. paraguariensis apresentou redução de $65,8 \%$ de sua área de adequabilidade climática, no cenário mais pessimista, a redução foi de $82,1 \%$, sendo as perdas concentradas nas áreas periféricas das projeções de ocorrência.
\end{abstract}

Palavras-chave: clima presente e futuro, erva-mate, modelagem de nicho ecológico, ocorrência geográfica.

\begin{abstract}
We modelled the climatic niche and evaluated the impact of climate changes on the potential geographic distribution of Ilex paraguariensis. This species has high social-cultural, economic and ecological importance in the southern portion of the Atlantic Forest. The occurrence areas were defined by the geographical coordinates of the localities where the species was recorded. Eight climatic variables were obtained from the WorldClim database. The species climatic niche modelling was performed using ten algorithms. The predictions of contemporary and future (2070) areas of climatic suitability were carried out from the consensus of TSS > 0.40 fits. For future predictions, an optimist (RCP4.5) and pessimist (RCP8.5) scenarios were considered, based on the fifth IPCC report. We observed that the species occurs predominantly in the Atlantic Domain. While in the optimistic scenario I. paraguariensis presented a reduction of $65.8 \%$ of its area of climatic suitability, in the most pessimistic scenario the reduction reached $82.1 \%$, being the range losses concentrated in peripheral areas of occurrence projections.
\end{abstract}

Key words: present and future climate, mate herb, ecological niche modelling, geographic occurrence.

\section{Introdução}

Entender a distribuição geográfica de espécies florestais é importante para subsidiar estratégias de conservação e de uso sustentável das mesmas, assim como para auxiliar na previsão dos impactos que poderão ser causados à vegetação natural devido às mudanças climáticas futuras (Higuchi et al. 2013). Isto ocorre porque todas as espécies apresentam exigências climáticas específicas, definindo, assim, os seus respectivos nichos ecológicos, que muitas vezes são filogeneticamente conservados (Manen et al. 2010; Morales et al. 2014). 
Destaca-se que, ao longo da história evolutiva do planeta, uma das respostas das espécies frente aos vários eventos de mudanças do clima, como os que ocorreram durante o Quaternário (Davis \& Shaw 2001), foi a alteração das suas distribuições geográficas. Normalmente, as espécies deixam de existir nos locais que passam a ter um clima inóspito, e passam a ocupar as áreas com clima favorável. Um padrão de resposta amplamente observado é o de mudanças da distribuição geográfica ao longo de gradientes latitudinais e altitudinais, de forma que esta dinâmica de clima $\mathrm{x}$ vegetação tem moldado paisagens naturais $\mathrm{e}$ definido a composição de espécies de diferentes regiões (Behling 2002). Porém, deve ser enfatizado que a influência das mudanças climáticas sobre as espécies é resultado de um processo complexo, não apenas relacionado à eficiência de dispersão ou migração, mas também das diferentes respostas a nível de organismos (genética, fisiológica e fenológica), de populações (dinâmica) e de comunidades (relações inter-específicas) (Bellard et al. 2012), de forma que a adaptação a novas condições ambientais não pode ser descartada.

No último século, as atividades de origem antrópica têm alterado profundamente o funcionamento dos ecossistemas em escala global (Crutzen 2006). Dentre as consequências mais evidentes do impacto das atividades antrópicas, estão as mudanças climáticas, que têm ocorrido em elevada velocidade no contexto da história evolutiva da Terra (Davis \& Shaw 2001), e que, associadas às alterações do uso do solo, tornam as respostas das espécies uma incógnita. Neste sentido, com o propósito de fomentar pesquisas que procuram avaliar o impacto das atividades humanas sobre a bioesfera, o Painel Intergovernamental sobre Mudanças Climáticas (IPCC), em seu quinto relatório de avaliação (AR5), apresentou diferentes cenários de mudanças climáticas chamados de Percursos de Concentração Representativos (RCPs) (Symon 2013). Enquanto que nos cenários otimistas espera-se grandes mudanças em termos de matriz energética e tecnológica, de forma a reduzir a emissão de gases de efeito estufa, no cenário mais pessimista assume-se que nenhuma política climática será adotada e ocorrerá o aumento populacional no planeta.

Diante deste contexto, a modelagem do nicho ecológico de espécies tem-se destacado como uma das principais ferramentas para a investigação da relação entre as espécies e o clima, permitindo análises que possam contribuir para a proteção e a conservação da biodiversidade, para a escolha das espécies vizando a restauração de ecossistemas (Gastón et al. 2014; López-Tirado \& Hidalgo 2016) e no entendimento dos padrões de distribuição geográfica em uma análise regional (Siqueira \& Durigan 2007; Rodrigues et al. 2015).

Como objeto de estudo, algumas espécies arbóreas, por apresentarem elevada importância econômica, social e ecológica, são de particular interesse para serem investigadas por meio desta abordagem, uma vez que as consequências de eventuais mudanças climáticas podem causar grande impacto sócio-econômico e ambiental. Dentre estas, destaca-se a erva-mate (Ilex paraguariensis A.St.-Hil.), que na porção sul da Floresta Atlântica tem grande significado sóciocultural, uma vez que a bebida produzida a partir de suas folhas, consumida na forma de chimarrão, é muito apreciada pela população, sendo considerada parte da identidade cultural da região. Do ponto de vista econômico, I. paraguariensis é um dos principais produtos não madereiros em áreas de Floresta Ombrófila Mista (Balzon et al. 2004), tendo sido amplamente explorado e utilizado pela indústria para a produção de diversos produtos, como bebidas, alimentos, cosméticos e produtos de higiene e de limpeza (Dallabrida et al. 2016). Em relação à sua importância ecológica, destaca-se o fato de I. paraguariensis, que é uma espécie dióica, polinizada por insetos das ordens Himenoptera, Coleoptera, Hemiptera e Diptera (Pires et al. 2014), ser uma das mais frequentes em áreas de Floresta Ombrófila Mista no Sul do Brasil (Jarenkow \& Budke 2009) e desempenhar importante papel como fonte de alimento para a avifauna (Colussi \& Prestes 2011).

Considerando o que foi até aqui exposto, o presente trabalho procurou investigar o nicho climático e o impacto de diferentes cenários de mudanças climáticas sobre a distribuição geográfica potencial de I. paraguariensis. Assumiu-se que esta espécie não apresenta limite de dispersão, uma vez que este tipo de abordagem pode produzir bons resultados (Engler et al. 2009), além dos frutos da mesma serem amplamente procurados pela avifauna, o que sugere uma boa dispersão. Com isso, espera-se gerar informações que possam ser relevantes para a conservação desta espécie em diferentes cenários de mudanças climáticas, a partir de inferências sobre as áreas que permanecerão com o clima propício ao desenvolvimento da mesma, consideradas refúgios climáticos, e as áreas que passarão a ter um clima inóspito. 


\section{Material e Métodos}

Os locais de ocorrência de Ilex paraguariensis foram obtidos a partir de coordenadas geográficas, que foram extraídas de trabalhos publicados na literatura científica (Suplemento Digital $<$ https:// figshare.com/s/246b6d526e35c3454f95 >) e de pontos de georreferenciamento de ocorrência da espécie do banco de dados SpeciesLink $(<\mathrm{http}: / /$ splink.cria.org.br/>), do Centro de Referência em Informação Ambiental (CRIA 2014). Ressalta-se que os dados utilizados são de domínio público, disponíveis de forma livre. Os dados de distribuição da espécie passaram por uma checagem rigorosa, para verificar a existência de dados duplicados e incoerências relacionadas à distribuição geográfica, provocadas, por exemplo, por erros de digitação de coordenadas, como proposto por Hijmans \& Elith (2015).

Como variáveis explicativas para a modelagem do nicho climático, foram consideradas 19 variáveis climáticas, que foram obtidas do banco de dados do WorldClim (<http://www.worldclim. org/>) (Hijmans et al. 2005), na resolução de 2,5 minutos (Suplemento Digital $<$ https://figshare.com/ s/246b6d526e35c3454f95>). Para fins de remoção de problemas relacionados à multicolinearidade das variáveis explicativas climáticas (Graham 2003), foram determinados os Fatores de Inflação da Variância (VIF) para as mesmas, sendo removidas aquelas altamente autocorrelacionadas entre si (VIF $>10$ ) (Dormann et al. 2013). O valor de VIF é dado por $1 /\left(1-r_{i}^{2}\right)$, sendo que $r_{i}^{2}$ representa o coeficiente de determinação da variável preditora, em relação às demais variáveis (Dormann et al. 2013).

Conforme sugerido por Varela et al. (2014), foi realizada uma filtragem ambiental dos pontos de ocorrência geográfica de Ilex paraguariensis. Segundo os mesmos autores, a filtragem ambiental é mais eficiente do que a filtragem espacial para aumentar a performance de modelos. Para isso, as variáveis climáticas explicativas sem multicolinearidade (VIF < 10) para cada local de ocorrência geográfica de I. paraguariensis foram ordenadas por meio de uma Análise de Componentes Principais (PCA). Em seguida, os scores nos dois primeiros eixos da ordenação, que explicam a maior parte da inércia total dos dados, foram extraídos para serem utilizados na filtragem ambiental. Este procedimento foi realizado por meio da função R envSample (Varela et al. 2014). Os dados ambientalmente filtrados passaram por uma conferência quanto à existência de ocorrências de espécimes cultivados, considerando o possível impacto da presença das mesmas na modelagem. Esta verificação se deu por meio da identificação de qualquer tipo de observação nos dados primários que fizesse referência que os espécimes fossem plantados ou cultivados.

Para a predição do impacto de mudanças climáticas no ano de 2070, com base no quinto relatório do IPCC, foram considerados os cenários: RCP4.5, por ser o cenário mais realista entre os três cenários mais otimistas, e o RCP8.5, por ser o mais pessimista. No cenário otimista (RCP4.5), assume-se a adoção, a nível global, de políticas de mitigação de emissão de gases causadores do efeito estufa. No cenário pessimista (RCP8.5), considerase que a humanidade não adotará estratégias para a redução das emissões dos gases causadores do efeito estufa e que estas continuarão crescentes. Para as projeções destes cenários, utilizou-se o modelo de circulação atmosférica HADGEM2-ES, do MET OFFICE do Reino Unido, que faz parte do Coupled Model Intercorporation Project Phase 5 (CMIP5). Os dados climáticos das projeções futuras foram obtidos do WorldClim (Hijmans et al. 2005).

Considerando que o resultado preditivo da modelagem pode ser influenciado pela natureza do algoritmo utilizado (Thuiller 2004), representando uma fonte de incerteza, optou-se por usar uma abordagem pluralista. Assim, foram testados diferentes algoritmos, com o propósito de buscar um consenso daqueles com maior qualidade preditiva (Thuiller 2004). Para isto, o nicho climático da espécie foi modelado por meio dos dez principais algoritmos utilizados na atualidade, sendo eles Modelo Linear Generalizado (GLM) (Guisan et al. 2002; Dobson \& Barnett 2008), Modelo de Impulsão Generalizado (GBM) (Freund \& Schapire 1997), Modelo Aditivo Generalizado (GAM) (Hastie \& Tibshirani 1990), Árvore de Classificação (CTA) (Breiman et al. 1984), Rede Neural Artificial (ANN) (Venables \& Ripley 2002), BIOCLIM (BC) (Busby 1991; Araujo \& Peterson 2012), Análise Discriminante Flexível (FDA) (Hastie et al. 1994), Regressão Adaptativa Multivariada por Splines (MARS) (Friedman \& Silverman 1989), Floresta Aleatória (RF) (Breiman 2003) e Máxima Entropia (Maxent) (Phillips et al. 2006). Para os modelos que requerem dados referentes a ausências, foram geradas 100 pseudoausências para cada observação da espécie (Lobo \& Tognelli 2011), que foram distribuídas, três vezes, de forma aleatória em um buffer com raio de 500 km (VanDerWal et al. 2009), no entorno de cada observação. Os dados foram subdivididos em dois 
grupos: uma parte composta por $70 \%$ dos dados para a calibração (treino) dos ajustes e os $30 \%$ restantes para a avaliação (teste) dos ajustes, conforme sugerido por Thuiller (2003). Para cada um dos dez algoritmos e das distribuições de pseudo-ausências, foram realizadas duas réplicas de calibração e teste, totalizando 60 ajustes (10 algoritmos $\times$ três distribuições de pseudo-ausências $\times$ duas réplicas de calibração e teste).

A performance dos modelos foi avaliada por meio da estatística TSS, que varia de - 1 a 1 (Allouche et al. 2006), sendo que o valor 1 representa um ajuste perfeito. Foram construídas curvas de respostas entre a estimativa de adequabilidade climática para a espécie (0-100) e as variáveis mais explicativas, de acordo com o modelo e o ajuste de melhor performance. Assim, com o propósito de reduzir as incertezas preditivas inerentes a cada modelo, foi determinada, a partir de um consenso dos ajustes com uma boa acurácia (TSS >0,40) (Landis \& Koch 1977), a estimativa de adequabilidade climática para a ocorrência de $I$. paraguariensis, tanto para o presente, quanto para o futuro (2070). O consenso foi construído por meio da média das estimativas de adequabilidade climática dos diferentes ajustes utilizados (TSS > 0,40). A projeção, contemporânea e futura, foi realizada para parte da América do Sul, com destaque para a porção sul e sudeste da Floresta Atlântica, que representa a maior parte da área de ocorrência natural da espécie. Além disso, foi quantificada a incerteza das projeções por meio da espacialização do coeficiente de variação. Para as projeções consensuais para o presente e o futuro, foram construídos mapas binários considerando áreas adequadas para a ocorrência da espécie aquelas cuja estimativa de adequabilidade tenha sido maior do que $50 \%$. Em seguida, foram determinadas a proporção de pixels (área de adequabilidade climática) perdidos, ganhos e estáveis para a espécie estudada, por meio da função BIOMOD_RangeSize, do pacote Biomod2 (Thuiller et al. 2014), da linguagem de programação estatística $\mathrm{R}$ ( $\mathrm{R}$ Core Team 2016). Todas as demais análises também foram realizadas no R, junto com os pacotes USDM (Naimi 2015), dismo (Hijmans et al. 2015), raster (Hijmans 2015) e biomod2 (Thuiller et al. 2014).

\section{Resultados}

A partir dos dados de ocorrência geográfica de Ilex paraguariensis, foi possível verificar que a espécie apresenta uma distribuição ampla no Brasil, podendo ser encontrado do Rio Grande do Sul até a Bahia, ocorrendo, inclusive, no
Brasil Central (Fig. 1a). Após a remoção de colinearidade entre as variáveis explicativas da área de adequabilidade climática da espécie, foram selecionadas oito variáveis climáticas,
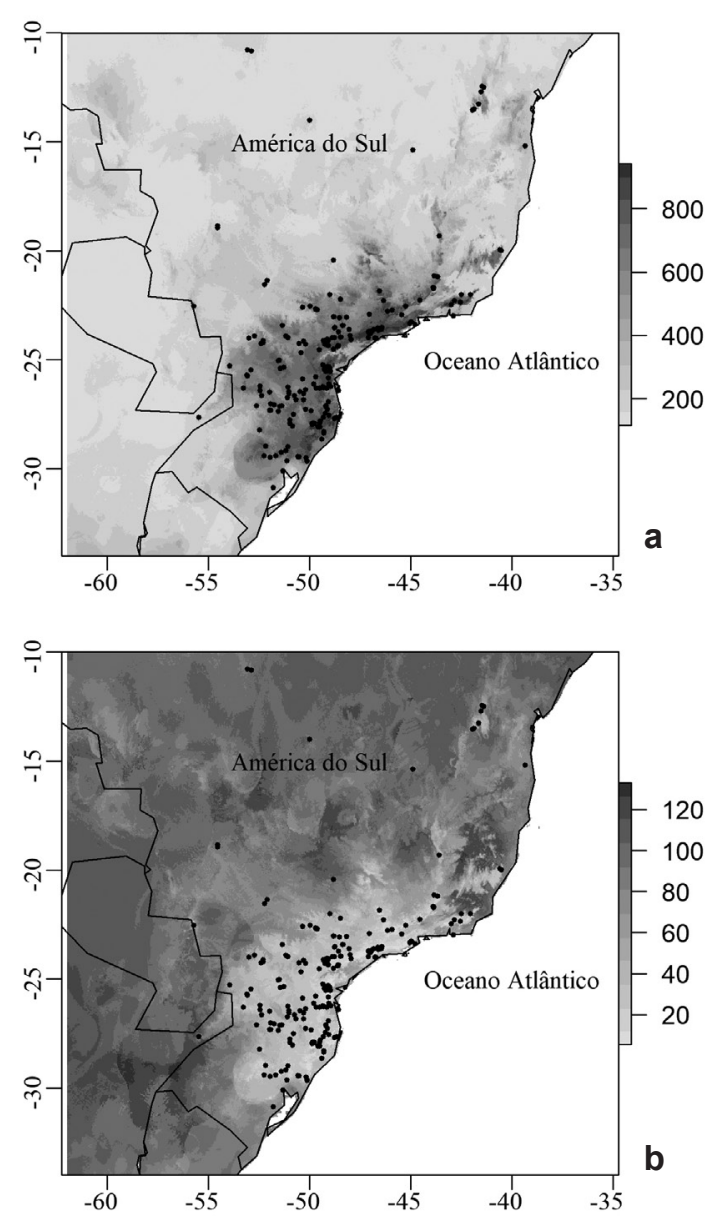

Figura 1 - a. Localização das ocorrências de Ilex paraguariensis, projeção da estimativa de adequabilidade climática. b. Projeção do coeficiente de variação da estimativa de adequabilidade climática, em função do consenso dos modelos com TSS > 0,40 . [Tons de cinza, de mais escuro para mais claro, representam o gradiente de maior a menor adequabilidade climática para a espécie (a) e de maiores a menores valores do coeficiente de variação para as estimativas de adequabilidade climática para a espécie (b)].

Figure 1 - a. Occurrence location of Ilex paraguariensis and projection of the estimated climate suitability. b. Projection of the coefficient of variation of the estimated climate suitability (b), according to the consensus of the models with TSS $>0.40$. [Gray shades, from darker to lighter, represent the gradient from high to low climatic suitability for the species (a) and the gradient from high to low values of coefficient of variation for species climatic suitability estimative (b)]. 
Tabela 1 - Variáveis bioclimáticas utilizadas como preditoras da distribuição geográfica de Ilex paraguariensis, após remoção de colinearidade. VIF = Fator de Inflação da Variância.

Table 1 - Variables used as predictors of the geographical distribution of Ilex paraguariensis, after removal of collinearity. VIF = Variance Inflation Factor.

\begin{tabular}{llc}
\hline Sigla & Variável & VIF \\
\hline bio2 & Média da amplitude térmica diária & 1,528030 \\
bio3 & Isotermalidade & 6,002698 \\
bio8 & Temperatura média do trimestre mais úmido & 6,725791 \\
bio9 & Temperatura média do trimestre mais seco & 5,828980 \\
bio10 & Temperatura média no trimestre mais quente & 8,110192 \\
bio12 & Precipitação anual & 1,720836 \\
bio18 & Precipitação do trimestre mais quente & 1,543085 \\
bio19 & Precipitação do trimestre mais frio & 2,898199 \\
\hline
\end{tabular}

sendo cinco relacionadas à temperatura (média da amplitude térmica diária, isotermalidade, temperatura média no trimestre mais úmido, temperatura média no trimestre mais seco e temperatura média no trimestre mais quente) e três relacionadas à precipitação pluviométrica (precipitação anual, precipitação no trimestre mais quente e precipitação no trimestre mais frio) (Tab. 1). Do total de pontos de ocorrências obtidos (306), após procedimentos de conferências e de filtragem ambiental, sobraram 111, que foram utilizados para as modelagens do nicho climático.
Dos 60 ajustes gerados para os dez algoritmos utilizados nas modelagens, 52 apresentaram uma boa performance $(\mathrm{TSS}>0,40)$. Apenas o algoritmo BIOCLIM produziu ajustes (quatro) com baixa performance (TSS <0,40) (Tab. 2).

As variáveis mais explicativas em relação à área de adequabilidade climática de $I$. paraguariensis para o modelo melhor ajustado $(\mathrm{GBM}$, com TSS $=0,76)$ foram, respectivamente, temperatura no trimestre mais quente e precipitação no trimestre mais frio. Ao se analisar as curvas de estimativa de adequabilidade climática em função

Tabela 2 - Quantidade de modelos (NM) ajustados para a distribuição geográfica de Ilex paraguariensis, em função de variáveis climáticas, com boa performance (TSS $>0,40$ ). Valores de TSS representam as médias dos ajustes com boa performance, com seus respectivos desvios-padrão (entre parênteses).

Table 2 - Number of models (NM) with good performance (TSS > 0.40) fitted in function of climatic variables for the geographic distribution of Ilex paraguariensis. TSS values represent the averages of the good performance fits, with their respective standard deviations (in parentheses).

\begin{tabular}{lcc}
\hline Algoritmos & NM & TSS \\
\hline Modelo Linear Generalizado (GLM) & 6 & $0,69(0,04)$ \\
Modelo de Impulsão Generalizado (GBM) & 6 & $0,70(0,05)$ \\
Modelo Aditivo Generalizado (GAM) & 6 & $0,60(0,07)$ \\
Árvore de Classificação (CTA) & 6 & $0,66(0,06)$ \\
Rede Neural Artificial (ANN) & 6 & $0,57(0,05)$ \\
BIOCLIM (BC) & 2 & $0,42(0,01)$ \\
Análise Discriminante Flexível (FDA) & 6 & $0,62(0,06)$ \\
Regressão Adaptativa Multivariada por Splines (MARS) & 6 & $0,66(0,06)$ \\
Floresta Aleatória (RF) & 6 & $0,62(0,06)$ \\
Máxima Entropia (MaxEnt) & 6 & $0,69(0,04)$ \\
\hline
\end{tabular}


das variáveis mais explicativas, observa-se que $I$. paguariensis apresentou distribuição geográfica associada a áreas cujo verão tenha temperaturas mais amenas $\left(<24^{\circ} \mathrm{C}\right)$ e inverno chuvoso $(>100$ mm) (Fig. 2).

A distribuição predita demonstrou que $I$. paraguariensis tem sua ocorrência geográfica
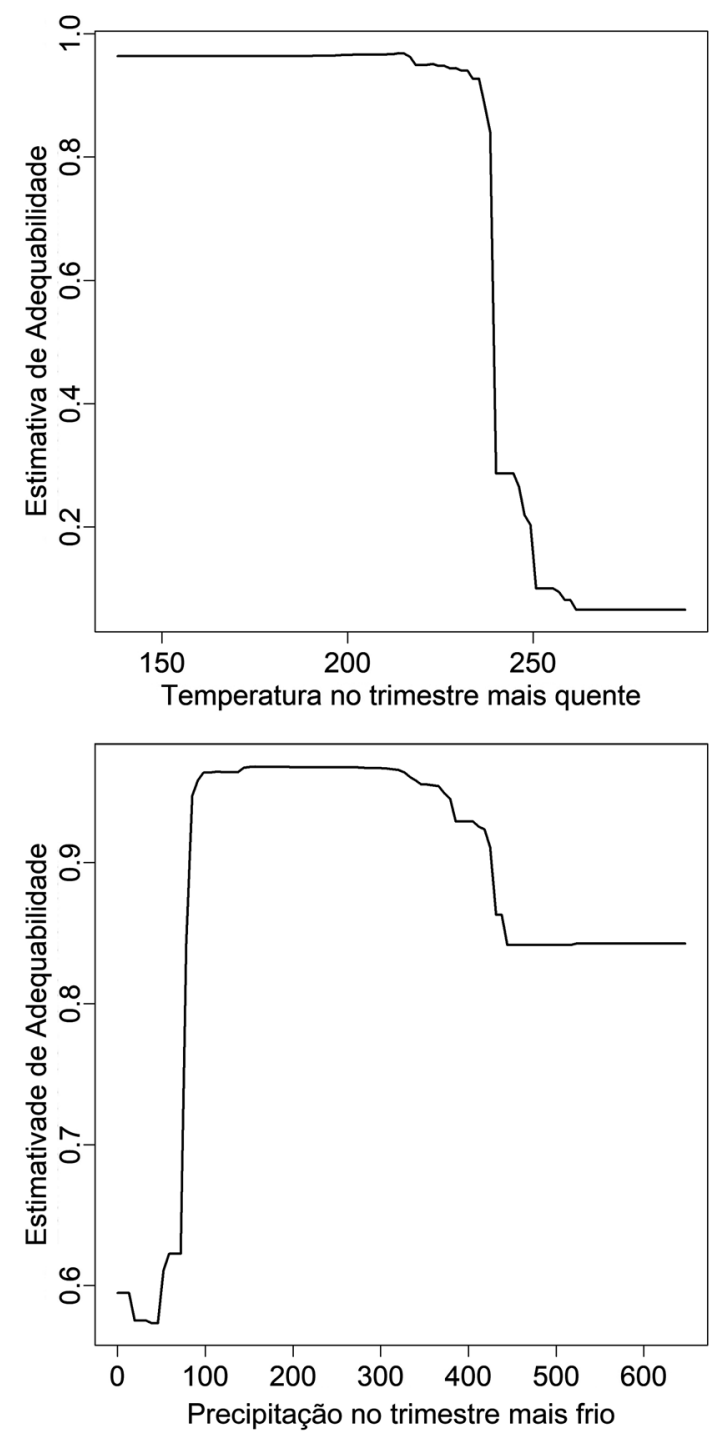

Figura 2 - Estimativa de adequabilidade climática para Ilex paraguariensis, ao longo dos gradientes climáticos de maior contribuição (temperatura no trimestre mais quente, em ${ }^{\circ} \mathrm{C} \times 10$, e precipitação no trimestre mais frio, em $\mathrm{mm}$ ), de acordo com o modelo melhor ajustado (GBM, com TSS =0,76).

Figure 2 - Climatic suitability estimative for Ilex paraguariensis along climatic gradients of greater contribution (mean temperature of warmest quarter, in ${ }^{\circ} \mathrm{C} \times 10$, and precipitation of coldest quarter, in $\mathrm{mm}$ ), according to the best fitted model (GBM with TSS $=0.76$ ). potencial que se sobrepõe, em grande parte, a porção sul e sudeste da Floresta Atlântica (Fig. 1a). Foi possível observar que a espécie apresenta uma elevada estimativa de adequabilidade climática nas áreas de maior elevação da região, representadas pelo Planalto Meridional, Serra Geral e Serra do Mar. Quanto à espacialização do coeficiente de variação para a predição consensual da adequabilidade climática (Fig. 1b), observa-se, de forma geral, valores baixos (cores mais claras), especialmente para a região sul e sudeste do Brasil, indicando elevada concordância dos ajustes utilizados para a construção do consenso.

Foi possível verificar uma mudança na distribuição geográfica potencial da espécie diante dos diferentes cenários de mudanças climáticas, em repostas ao seu nicho climático (Fig. 3). As perdas das áreas com adequabilidade ocorreram, predominantemente, de forma periférica, concentradas nos locais de menor altitude. As áreas de elevada altitude das regiões Sul e Sudeste do Brasil permaneceram com adequabilidade climática, caracterizando-se como áreas estáveis. Já as áreas que passaram a apresentar adequabilidade climática foram praticamente inexistentes para ambos os cenários $(\mathrm{RCP} 4.5=0,7 \%$; RCP8.5 $=$ $0,0 \%$ ). Desta forma, observa-se uma redução estimada de $65,8 \%$ e $82,1 \%$ da área de distribuição potencial diante, respectivamente, do cenário otimista (RCP4.5) e pessimista (RCP8.5) de mudanças climáticas.

\section{Discussão}

Os resultados demonstraram que a espécie ocorre em domínio predominantemente Atlântico, com uma distribuição ampla em sua porção sul e sudeste. De fato, estudos têm demonstrado uma distribuição ampla para Ilex paraguariensis, que foi classificado como uma espécie comum para a Floresta Atlântica Úmida, entre o Espírito Santo e Rio Grande do Sul, por Caiafa \& Martins (2010). Desta forma, a projeção contemporânea das estimativas de adequabilidade climática, a partir da modelagem realizada para a espécie, está coerente com os padrões descritos na literatura.

Variáveis climáticas que indicam sazonalidade e fatores ambientais extremos ou limitantes, como a temperatura no trimestre mais quente e a precipitação no trimestre mais frio, demonstraram ser importantes elementos ecológicos na determinação da área de ocorrência de I. paraguariensis. De forma geral, os resultados sugerem que a espécie tem um caráter não tropical 
e é intolerante à seca no inverno, o que pode explicar sua preferência de ocorrência no Sul e Sudeste do Brasil.

Destaca-se que o ancestral mais antigo de todas as espécies de Ilex existiu no Hemisfério
Norte (América do Norte ou Leste Asiático), durante o Mioceno, onde, a partir de vários eventos de dispersão, o gênero se dispersou para outras partes do mundo (Manen et al. 2010). Na América do Sul, Ilex passou por um processo
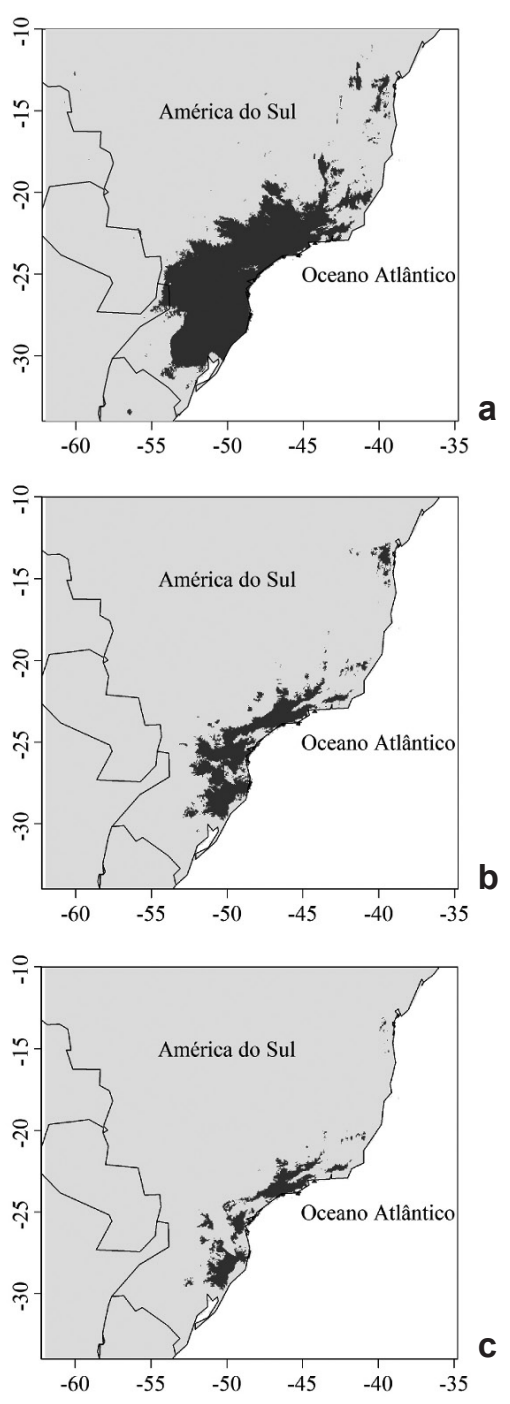
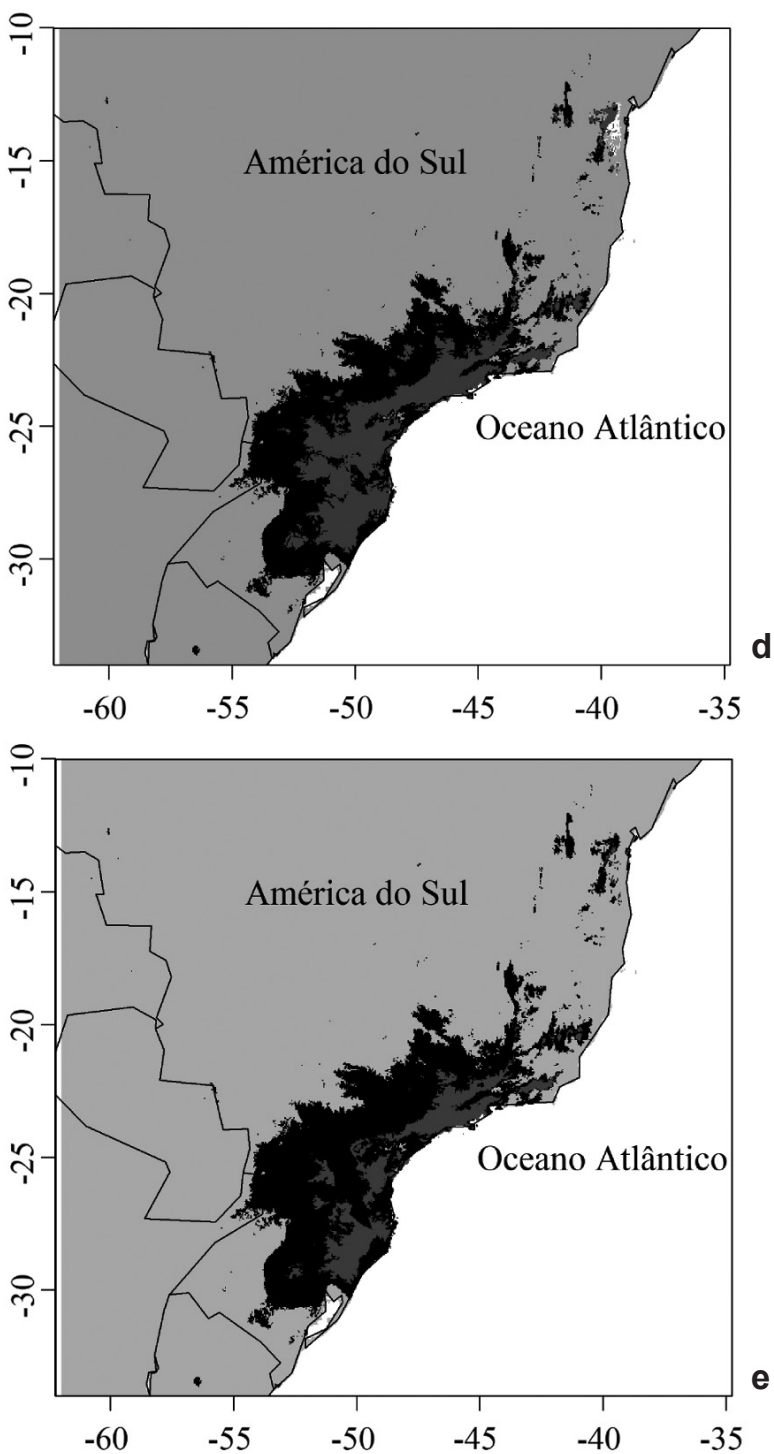

Figura 3 - Áreas com clima favorável (cinza escuro) à ocorrência de Ilex paraguariensis para o presente (a) e para o futuro (2070), de acordo com os cenários RCP4.5 (b) e RCP8.5 (c) do IPCC, com indicação dos padrões de mudanças, conforme cenários otimista $(\mathrm{d})$ e pessimista $(\mathrm{e})$. Cinza escuro (d, e): áreas estáveis, que apresentam o clima favorável no presente e continuarão apresentando a adequabilidade climática no futuro; cinza claro (d, e): áreas estáveis, que não apresentam o clima favorável e continuarão não apresentando no futuro; preto (d, e): áreas instáveis, que deixarão de apresentar condições favoráveis para a ocorrência da espécie; branco (d): áreas instáveis, que passarão a apresentar condições climáticas favoráveis. Figure 3 - Areas with suitable climate (dark gray) for Ilex paraguariensis occurrence in the present (a) and in the future (2070), according to IPCC RCP4.5 (b) and RCP8.5 scenarios (c), indicating the change patterns, according optimist (d) and pessimist (e) scenarios. Dark gray (d, e): stable areas which have suitable climate and will continue presenting climatic suitability in the future, light gray (d, e): stable areas, which do not have suitable climate and will not present in the future; Black (d, e): unstable areas, which will no longer provide favorable conditions for the occurrence of the species; White (d): unstable areas, which will provide favorable climatic conditions. 
de grande diversificação, de forma que a região é considerada, junto com o Leste Asiático, a área de maior diversidade do gênero (Cuénoud et al. 2000). I. paraguariensis apresenta maior proximidade filogenética com espécies de ocorrência no sudeste norte-americano (Ilex vomitoria Sol. ex Aiton), nas montanhas da América Central (Ilex tolucana Hemsley) e nos Andes colombiano (Ilex laurina Kunth), que com espécies do leste da América do Sul (e.g., Ilex microdontha Reissek, Ilex theezans Mart. ex Reissek e Ilex brevicuspis Reissek) (Cuénoud et al. 2000). Este padrão sugere que as espécies do gênero apresentam elevada capacidade de dispersão e baixo isolamento reprodutivo (Manen et al. 2010).

Além de fatores ecológicos, não se pode ignorar a capacidade da influência humana sobre o padrão de distribuição geográfica das espécies arbóreas. Neste contexto, Reis et al. (2014) e Bitencourt \& Krauspenhar (2006) argumentam que a Floresta Ombrófila Mista, na qual $I$. paraguariensis é uma das espécies mais frequentes, representa uma formação florestal que tem sido influenciada por práticas antrópicas há milênios, de forma que a sua área de ocorrência natural pode ser considerada como uma paisagem cultural. No caso de I. paraguariensis, este é um aspecto que potencialmente apresenta grande importância, uma vez que o seu uso como estimulante, medicinal e xamânico foi uma prática comum entre os povos pré-colombianos (Klanovicz et al. 2008; Contini et al. 2012), o que pode estar relacionado com a sua distribuição mais ampla.

Quanto ao impacto dos diferentes cenários de mudanças climáticas sobre a distribuição geográfica potencial de I. paraguariensis, o presente estudo demonstrou uma tendência de alterações ao longo de gradientes altitudinais, de forma que locais de menor altitude deixarão de ter condições mais favoráveis à sua ocorrência. Estudos têm demostrado que este processo ocorre atualmente de forma muito rápida, em função de mudanças climáticas recentes (Hickling et al. 2006; Kelly \& Goulden 2008; Lenoir et al. 2008; Chen et al. 2011). Estas circunstâncias, associadas a outros fatores de natureza antrópica, como a alteração do uso solo, são inéditas no planeta (Davis \& Shaw 2001), o que aumenta a incerteza quanto às predições futuras de ocorrência geográfica de espécies.

Apesar de Lenoir et al. (2008) terem verificado na Europa que o impacto das mudanças climáticas em espécies vegetais atinge tanto as áreas marginais quanto as áreas centrais de ocorrência, no presente estudo foi observada a concentração de perdas apenas nas áreas marginais, de forma que os padrões de respostas podem ser dependentes de aspectos relacionados à geografia da região avaliada (e.g., relevo, continentalidade, latitude). Como na região de estudo a espécie avaliada já ocupa pisos altitudinais elevados, observou-se que as áreas com adequabilidade climática ficarão restritas aos de locais de maior altitude. Estes resultados sugerem o potencial de áreas de maior altitude em funcionarem como refúgios climáticos para diversas espécies.

$\mathrm{O}$ presente estudo reforça a ideia de que a elaboração de planos para a proteção da biodiversidade frente às mudanças do clima deve se tornar prioritária em países megadiversos, como o Brasil. Em se tratando de Ilex paraguariensis, uma espécie da Mata Atlântica de grande importância sociocultural, econômica e ecológica, fica evidente a necessidade de adoção de estratégias que tenham com propósito a mitigação dos possíveis impactos de mudanças climáticas. Com base no trabalho de Hoegh-Guldberg et al. (2008), destacam-se as seguintes possibilidades: i) a manutenção, a expansão e a criação de novas Unidades de Conservação em áreas estratégicas, que permanecerão com boa adequabilidade climática, como aquelas situadas nos locais de maior altitude; ii) melhorar a conectividade da paisagem, para facilitar o fluxo da espécie para áreas que funcionarão como refúgios climáticos; iii) a utilização de técnicas de melhoramento genético, para a seleção de variedades mais tolerantes às condições climáticas futuras; iv) o resgate de populações de áreas que passarão a ter clima inadequado, para a conservação ex-situ; e v) a dispersão assistida, por meio de transferência de propágulos para áreas que permanecerão com adequabilidade climática.

Conclui-se que I. paraguariensis apresenta uma distribuição geográfica associada, principalmente, ao Domínio Atlântico, e com um caráter subtropical e temperado úmido. As áreas de maior altitude da região Sul e Sudeste do Brasil podem ser consideradas como estratégicas para a conservação e manejo sustentável da espécie, uma vez que apresentaram os maiores valores de estimativa de adequabilidade climática para $I$. paraguariensis e podem funcionar como refúgios climáticos em um cenário de aquecimento global. Ressalta-se, ainda, que, predominantemente em 
regiões periféricas de sua ocorrência, é predita elevada perda das áreas com adequabilidade climática, de forma que estas são prioritárias para os resgaste de populações, para a criação de bancos de germoplasma, com intuito de preservação da variabilidade genética da espécie. Nos cenários otimista e pessimista de mudanças climáticas, estima-se uma redução, respectivamente, de 65,8 a $82,1 \%$ da área de sua ocorrência geográfica potencial.

\section{Material suplementar}

O suplemento digital disponível em $<$ https:// figshare.com/s/246b6d526e 35c3454f95> contém uma listagem de trabalhos utilizados para a extração das coordenadas geográficas de Ilex paraguariensis.

\section{Agradecimentos}

À FAPESC (Fundação de Amparo à Pesquisa e Inovação do Estado de Santa Catarina), a concessão de bolsa de Mestrado em Engenharia Florestal à primeira autora do trabalho. Ao CNPq, a concessão de bolsa de Produtividade para o segundo e o terceiro autores e apoio financeiro por meio do Edital CTI/CNPQ/Universal 14/2014.

\section{Referências}

Allouche O, Tsoar A \& Kadmon R (2006) Assessing the accuracy of species distribution models: prevalence, kappa and the true skill statistic (TSS). Journal of Applied Ecology 43: 1223-1232.

Araujo MB \& Peterson AT (2012) Uses and misuses of bioclimatic envelope modeling. Ecology 93: 1527-1539.

Balzon DR, Silva JCGL \& Santos AJ (2004) Aspectos mercadológicos de produtos florestais não madereiros - análise retrospectiva. Revista Floresta 34: 363-371.

Behling H (2002) South and southeast Brazilian grasslands during Late Quaternary times: a synthesis. Palaeogeography, Palaeoclimatology, Palaeoecology 177: 19-27.

Bellard C, Bertelsmeier C, Leadley P, Thuiller W \& Courchamp F (2012) Impacts of climate change on the future of biodiversity. Ecology letters 15: 365-377.

Bitencourt ALV \& Krauspenhar PM (2006) Possible prehistoric anthropogenic effect on Araucaria angustifolia (Bert.) O.Kuntze expansion during the late holocene. Revista Brasileira de Paleontologia 9: 109-116.

Breiman L (2003) Manual on settings up, using and understanding random forest. Vol. 4. University of California Berkeley, Statistics Department, Berkeley. 33p.

Breiman L, Friedman JH, Olshen RA \& Stone CJ (1984) Classification and regression trees. Wadsworth and Brooks/Cole, Monterey. 358p.

Busby JR (1991) BIOCLIM - a bioclimatic analysis and prediction system. In: Margules CR \& Austin MP (eds.) Nature conservation: cost effective biological surveys and data analysis. CSIRO, Canberra. Pp. 64-68.

Caiafa AN \& Martins FR (2010) Forms of rarity of tree species in the southern Brazilian Atlantic rainforest. Biodiversity and Conservation 19: 2597-2618.

Chen IC, Hill JK, Ohlemüller R, Roy DB \& Thomas CD (2011) Rapid range shifts of species associated with high levels of climate warming. Science 333: 1024-1026.

Colussi J \& Prestes NP (2011) Frugivoria realizada por aves em Myrciaria trunciflora (Mart.) O. Berg. (Myrtaceae), Eugenia uniflora L. (Myrtaceae) e Ilex paraguariensis St. Hil. no norte do estado do Rio Grande do Sul. Revista Brasileira de Ornitologia 19: $48-550$

Contini AZ, Castilho MA \& Costa RB (2012) A erva-mate e os Kaiowá e Guarani: da abordagem etnobotânica à promoção do desenvolvimento local. Interações 13: 161-168.

CRIA (2014) SpeciesLink. Disponível em $<$ http://splink. cria.org.br/>. Acesso em 9 junho 2014.

Crutzen PJ (2006) The "Anthropocene". In: Ehlers E \& Krafft T (eds.) Earth System Science in the Anthropocene, New York. Pp. 13-18.

Cuénoud P, Martinez MADP, Loizeay PA, Spichiger R, Andrews S \& Manen JF (2000) Molecular phylogeny and biogeography of the genus Ilex L. (Aquifoliaceae). Annals of Botany 85: 111-122.

Dallabrida VR, Dumke CI, Molz S, Furini V \& Giacomelli MBO (2016) Com erva-mate não se faz só chimarrão! Situação atual e perspectiva de inovação no setor ervateiro do planalto norte catarinense. Revista Eletronica do Programa de Mestrado em Desenvolvimento Regional da Universidade do Contestado 6: 247-273.

Davis MB \& Shaw RG (2001) Range shifts and adaptive responses to quaternary climate change. Science 292: 673-679.

Dobson AJ \& Barnett A (2008) An introduction to generalized linear models. $3^{\text {rd }}$ ed. Chapman and Hall, London. 320p.

Dormann CF, Elith J, Bache S, Buchmann C, Carl G, Carré G, Marquéz JRG, Gruber B, Lafourcade B, Leitão PJ, Münkemüller T, McClean C, Osborne PE, Reineking B, Schröeder B, Skidmore AK, Zurell D \& Lautenbach S (2013) Collinearity: a review of methods to deal with it and a simulation study evaluating their performance. Ecography 36: 27-46.

Engler R, Randin CF, Vittoz P, Czáka T, Beniston M, Zimmermann NE \& Guisan A (2009) Predicting 
future distributions of mountain plants under climate change: does dispersal capacity matter? Ecography 32: 34-45.

Freund Y \& Schapire RE (1997) A decision-theoretic generalization of on-line learning and an application to boosting. Journal of Computer and System Sciences 55: 119-139.

Friedman JH \& Silverman BW (1989) Flexible parsimonious smoothing and additive modeling. Technometrics 31: 3-21.

Gastón A, García-Viñas JI, Bravo-Fernández AJ, LópezLeiva C, Oliet JA, Roig S \& Serrada R (2014) Species distribution models applied to plant species selection in forest restoration: are model predictions comparable to expert opinion? New forests 45 : 641-653.

Graham MH (2003) Confronting multicollinearity in ecological multiple regression. Ecology 84: 28092815.

Guisan A, Edwards Jr TC \& Hastie T (2002) Generalized linear and generalized additive models in studies of species distributions: setting the scene.Ecological Modelling 157: 89-100

Hastie T \& Tibshirani R (1990) Generalized Additive Models. Chapman and Hall, London. 352p.

Hastie T, Tibshirani R \& Buja A (1994) Flexible discriminant analysis by optimal scoring. Journal of the American statistical association 89: 1255-1270.

Hickling R, Roy DB, Hill JK, Fox R \& Thomas CD (2006) The distributions of a wide range of taxonomic groups are expanding polewards. Global Change Biology 12: 450-455.

Higuchi P, Silva AC, Budke JC, Mantovani A, Bortoluzzi RLC \& Ziger AA (2013) Influência do clima e de rotas migratórias de espécies arbóreas sobre o padrão fitogeográfico de florestas na região sul do Brasil. Ciência Florestal 23: 539-553.

Hijmans RJ, Cameron SE, Parra JL, Jones PG \& Jarvis A (2005) Very high resolution interpolated climate surfaces for global land areas. International Journal of Climatology 25: 1965-1978.

Hijmans RJ (2015) raster: geographic data analysis and modeling. R package version 2.3-40. Disponível em $<$ https://cran.r-project.org/package=raster $>$. Acesso em 27 agosto 2016.

Hijmans RJ \& Elith J (2015) Species distribution modeling with R. Disponível em $<$ https://cran.rproject.org/web/packages/dismo/vignettes/sdm. pdf $>$. Acesso em 27 agosto 2016.

Hijmans RJ, Phillips S, Leathwick J \& Elith J (2015) dismo: species distribution modeling. R package version 1.0-12. Disponível em <https://cran.rproject.org/package $=$ dismo $>$. Acesso em 27 agosto 2016.

Hoegh-Guldberg O, Hughes L, McIntyre S, Lindenmayer DB, Parmesan C, Possingham HP \& Thomas CD (2008) Assisted colonization and rapid climate change. Science 321: 345-346.
Jarenkow JA \& Budke JC (2009) Padrões florísticos e análise estrutural de remanescentes florestais com Araucaria angustifolia no Brasil. In: Fonseca CSD, Souza AF, Zanchet AML, Dutra T, Backes A \& Ganade GMS (eds.) Floresta com Araucária: ecologia, conservação e desenvolvimento sustentável. Holos, Ribeirão Preto. Pp. 113-126.

Kelly AE \& Goulden ML (2008) Rapid shifts in plant distribution with recent climate change. Pnas 105: 11.823-11.826.

Klanovicz J, Fert Neto J \& Mafra AL (2008) Southern brazilian indigenous populations and the forest: towards an environmental history. Ekonomska i Ekohistorija 4: 94-103.

Landis JR \& Koch GG (1977) The measurement of observer agreement for categorical data. Biometrics 33: 159-174. DOI: <http://dx.doi. org/10.2307/2529310>.

Lenoir J, Gégout JC, Marquet PA, de Ruffray P \& Brisse $\mathrm{H}$ (2008) A significant upward shift in plant species optimum elevation during the $20^{\text {th }}$ century. Science 320: 1768-1771.

Lobo JM \& Tognelli MF (2011) Exploring the effects of quantity and location of pseudo-absences and sampling biases on the performance of distribution models with limited point occurrence data. Journal for Nature Conservation 19: 1-7.

López-Tirado J \& Hidalgo PJ (2016) Ecological niche modelling of three Mediterranean pine species in the south of Spain: a tool for afforestation/reforestation programs in the twenty-first century. New forests 47: 411-42

Manen JF, Barriera G, Loizeau PA \& Naciri Y (2010) The history of extant Ilex species (Aquifoliaceae): evidence of hybridization within a Miocene radiation. Molecular phylogenetics and evolution 57: 961-977.

Morales M, Wulff AF, Fortunato RH \& Poggio L (2014) Chromosome studies in southern species of Mimosa (Fabaceae, Mimosoideae) and their taxonomic and evolutionary inferences. Plant Systematics and Evolution 300: 803-817.

Naimi B (2015) usdm: uncertainty analysis for species distribution models. R package version 1.115. Disponível em <https://cran.r-project.org/ package $=$ usdm $>$. Acesso em 27 agosto 2016.

Phillips SJ, Anderson RP \& Schapire RE (2006) Maximum entropy modeling of species geographic distributions. Ecological modelling 190: 231-259.

Pires EZ, Stedille LIB, Machado S, Mantovani A \& Bortoluzzi RLC (2014) Biologia reprodutiva de erva-mate (Ilex paraguariensis A.St. Hill.) em remanescentes de Floresta Ombrófila Mista Altomontana. Revista de Ciências Agroveterinárias 13: $171-180$.

R Core Team (2016) R: a language and environment for statistical computing. R Foundation for 
Statistical Computing. Disponível em $<$ https:// www.r-project.org/>. Acesso em 27 agosto 2016.

Reis MS, Ladio A \& Peroni N (2014) Landscapes with Araucaria in South America: evidence for a cultural dimension. Ecology and Society 19: 43.

Rodrigues PMS, Silva JO, Eisenlohr PV \& Schaefer CEGR (2015) Climate change effects on the geographic distribution of specialist tree species of the Brazilian tropical dry forests. Brazilian Journal of Biology 75: 679-684.

Siqueira MF \& Durigan G (2007) Modelagem da distribuição geográfica de espécies lenhosas de cerrado no estado de São Paulo. Revista brasileira de Botânica 30: 233-243.

Symon CC (2013) Mudanças do clima: ação, tendências e implicações para o mundo empresarial. Quinto relatório de avaliação do IPCC, grupo de trabalho 1. Universidade de Cambridge. Disponível em $<$ http://www.cnseg.org.br/lumis/portal/file/ fileDownload.jsp?fileId=485C88E7424C61FD01 42DDCA73D41743>. Acesso em 27 agosto 2016.

Thuiller W (2003) BIOMOD - optimizing predictions of species distributions and projecting potential future shifts under global change. Global Change Biology 9: 1353-1362.

Thuiller W (2004) Patterns and uncertainties of species' range shifts under climate change. Global Change Biology 10: 2020-2027.

Thuiller W, Georges D \& Engler R (2014) biomod2: Ensemble platform for species distribution modeling. R package version 3.1-64. Disponível em <https://cran.r-project.org/web/packages/ biomod2/index.html $>$. Acesso em 27 agosto 2016.

Varela S, Anderson RP, García-Valdés R \& FernándezGonzález F (2014) Environmental filters reduce the effects of sampling bias and improve predictions of ecological niche models. Ecography 37: 1084-1091.

VanDerWal J, Shoo LP, Graham C \& Williams SE (2009) Selecting pseudo-absence data for presence-only distribution modeling: how far should you stray from what you know? Ecological modelling 220: 589-594.

Venables WN \& Ripley BD (2002) Modern applied statistics with S. $4^{\text {th }}$ ed. Nova York, Springer. $495 p$. 